\title{
Graded epidural anesthesia for open cholecystectomy in elderly patient with ischemic heart disease
}

\author{
Shirin Parveen ${ }^{1}$, Supriya Singh ${ }^{2}$ \\ From ' Assistant Professor, 'Junior Resident, Department of Anaesthesia, Era's Lucknow Medical College and Hospital, Hardoi Road, Sarfarazganj, \\ Lucknow, India
}

\begin{abstract}
Geriatric patients with a limited physiological reserve and associated comorbidities present a challenge to anesthesiologists. Patients with cardiac disease coming for non-cardiac surgical procedures are at increased risk of perioperative cardiovascular events such as myocardial ischemia, myocardial infarction (MI) and are associated with greater morbidity and mortality. In this case report, the patient had a previous history of MI and was a known case of diabetes mellitus with acute cholecystitis with cholelithiasis with anterolateral ischemic heart disease with an ejection fraction of $35 \%$. This patient was posted for open cholecystectomy with CBD exploration and successfully managed under graded epidural anesthesia and had an uneventful recovery.
\end{abstract}

Key words: Geriatric anesthesia, Graded epidural anesthesia, Ischemic heart disease

I schemic heart disease (IHD) is a leading cause of morbidity and mortality in the perioperative period in cardiac patients [1]. The incidence of perioperative complications is far higher among patients with recent myocardial infarction (MI) or unstable angina who require urgent or emergency noncardiac surgery $[2,3]$. The primary goal of anesthesia in these patients is to avoid ischemia by balancing myocardial oxygen demand and supply. A thorough pre-operative assessment of risk factors, cardiovascular examination, pre-operative evaluation and optimization, monitoring, and an appropriate anesthetic plan are required for patients with ischemic heart disease. Various risk factors influencing perioperative cardiac morbidity are recent myocardial infarction, congestive cardiac failure, peripheral vascular disease, unstable angina, diabetes mellitus, hypertension, hypercholesterolemia, dysrhythmias, old age, renal dysfunction, obesity, and smoking. Elderly people with poor cardiac reserve undergoing upper abdominal surgery have a higher incidence of morbidity and mortality. Myocardial ischemia is precipitated when there is an imbalance between coronary blood flow and myocardial oxygen consumption. Prevention of a perioperative myocardial infarction is important to improve overall post-operative outcomes.

Graded Epidural Anesthesia (GEA) can decrease cardiac morbidity and mortality in ischemic heart disease (IHD) patients

\section{Access this article online}

Received - 25 August 2021

Initial Review - 11 September 2021

Accepted - 30 September 2021

DOI: $10.32677 /$ ijcr.v7i10.3085 posted for non-cardiac surgery. There are very few case reports on GEA for IHD patients coming for non-cardiac surgery as there is a higher rate of perioperative cardiac complications in such patients. Here, we report successful anesthetic management of a patient with acute cholecystitis with cholelithiasis with anterolateral IHD with ejection fraction $35 \%$, posted for open cholecystectomy with common bile duct (CBD) exploration under GEA.

\section{CASE REPORT}

A 72-year-old female with a known history of IHD, hypertension, and type-II diabetes for the last 5 years was diagnosed to have acute cholecystitis with cholelithiasis and was posted for open cholecystectomy with CBD exploration. The patient had undergone angioplasty 2 years back and was on Tab. Metoprolol-25 mg OD, Tab. nitroglycerine-2.6 mg BD, Tab. Aspirin-75 mg OD, Tab. Clopidogrel-75 mg OD, and Tab. Metformin-500mg OD. Of these, aspirin was continued and clopidogrel was discontinued 7 days before surgery. After 7 days, Prothrombin Time-International Normalized Ratio (PT-INR) was done and found normal. The patient was shifted from oral hypoglycemic agents to regular insulin according to a sliding scale before surgery.

On general examination, the patient was averagely built with a non-invasive blood pressure of $150 / 90 \mathrm{mmHg}$, a pulse rate of $90 / \mathrm{min}$, respiratory rate of $16 / \mathrm{min}$, and temperature of $98.6^{\circ}$ Fahrenheit. She was partially edentulous with adequate mouth opening and Mallampatti Grade-II. She had normal neck and

Correspondence to: Shirin Parveen, Department of Anesthesia, Era's Lucknow Medical College and Hospital, Hardoi Road, Sarfarazganj, Lucknow, India. E-mail: shirin_grmc@yahoo.com

(C) 2021 Creative Commons Attribution-NonCommercial 4.0 International License (CC BY-NC-ND 4.0). 
temporomandibular joint movements. The spine examination was normal. She was New York Heart Association-II (NYHA) and her breath-holding time was $<20 \mathrm{~s}$. On auscultation, the first and second heart sounds were audible with no added sound. On auscultation of the chest, bilateral vesicular breath sounds were heard with no added sounds.

Her routine laboratory investigations revealed Hb-11.0 g/dl, total leukocyte count (TLC)-6000 cells/cumm, platelet count-1.6 lakh, urea- $16 \mathrm{mg} / \mathrm{dl}$, creatinine- $0.8 \mathrm{mg} / \mathrm{dl}$, sodium- $137 \mathrm{mmol} / \mathrm{L}$, potassium-3.7 mmol/L, and serum bilirubin-2.5 mg/dl. Chest X-ray was within normal limits. Electrocardiography (ECG) showed anterolateral ischemic changes. Echocardiography showed severe hypokinesia of the septum and anterior wall with compromised left ventricular systolic function, IHD, mild mitral regurgitation (MR), mild tricuspid regurgitation (TR), and ejection fraction of $35 \%$. The cardiologist's opinion was obtained (Class III cardiac risk index).

The patient was accepted under the American Society of Anesthesiologists (ASA) Grade III with high risk. After preoperative counseling and written and informed consent, oral Alprazolam $0.25 \mathrm{mg}$ was given the night before surgery. Antihypertensive drugs and beta-blockers were continued on the day of surgery. Her fasting blood glucose was $120 \mathrm{mg} / \mathrm{dL}$.

After securing a18G IV access, injection Midazolam $0.5 \mathrm{mg}$ was given along with injection Ondansetron $4 \mathrm{mg}$. All the monitors- $\mathrm{SpO}_{2}, \mathrm{ECG}$, and non-invasive blood pressure (NIBP) were attached. Epidural catheter placement was performed under aseptic precautions in a sitting position. The skin was infiltrated with $2 \%$ plain lignocaine. Using an $18 \mathrm{G}$ Tuohy's needle, epidural space was confirmed at T9-T10 intervertebral space with loss of resistance technique. 20G epidural catheter was threaded and fixed at $9 \mathrm{~cm}$. Test dose was given with $3 \mathrm{ml}$ of $2 \%$ lignocaine with adrenaline after negative aspiration of cerebrospinal fluid (CSF) and blood in the sitting position. $3-5 \mathrm{ml}$ aliquots of plain bupivacaine $0.5 \%$ were given through the catheter. A total of $15 \mathrm{ml}$ of the drug was given. The blockade was obtained up to the level of T6. Supplemental oxygen was given through a face mask. The gall bladder was removed uneventfully and CBD exploration was done with T-tube insertion. The duration of surgery was $80 \mathrm{~min}$. Total intraoperative IV fluid (normal saline) given was $900 \mathrm{ml}$. The intraoperative urine output was $180 \mathrm{ml}$ and estimated blood loss was $150 \mathrm{ml}$. The patient had two episodes of hypotension: One at $10 \mathrm{~min}$ and the other at $45 \mathrm{~min}$ after the block and it was managed by giving an injection of Ephedrine $5 \mathrm{mg}$ each. The heart rate was maintained between 60 and 90/min. There was no episode of bradycardia, arrhythmia, or shoulder pain. Post-operative SpO2, NIBP, and ECG monitoring with oxygen supplementation through a face mask were performed in the Post Anesthesia Care Unit (PACU).

The patient remained hemodynamically stable in the post-operative period. Top-up doses of $6 \mathrm{ml}$ of $0.125 \%$ plain bupivacaine were given on the patient's request for post-operative analgesia. The epidural catheter was removed on the $3^{\text {rd }}$ day postoperatively and the patient was shifted to the surgical ward from where she was discharged after 1 week. Her stay in the hospital was uneventful.

\section{DISCUSSION}

Cardiovascular complications account for $25-50 \%$ of deaths following non-cardiac surgery. Patient characteristics, the sort of surgery proposed, and therefore the potential anesthetic risks have an impact on anesthetic choice and perioperative management. The anesthetic goals in managing patients with IHD are maintaining a favorable myocardial oxygen supply-demand relationship, stable hemodynamics, prevent perioperative MI, monitor for ischemia, maintain normothermia, and avoid significant blood loss. Surgery with its associated trauma and anesthesia can initiate inflammatory, hypercoagulable stress, and hypoxic state associated with perioperative elevation of troponin levels, arterial thrombosis, and mortality. In patients with cardiovascular disease, regional anesthesia techniques can offer the potential perioperative benefits of stress response attenuation, cardiac sympathectomy, shorter hospital stay, and intense postoperative analgesia. Thoracic epidural anesthesia has been found in many studies to vasodilate stenotic coronaries, thus reducing the number and duration of cardiac ischemic episodes $[4,5]$. It reduces 0 -to 30 day mortality and decreases the risk of pneumonia [6].

In this case, the patient had a previous history of MI and was a known case of diabetes mellitus. All anesthetic techniques must aim to avoid ischemia by keeping myocardial oxygen supply more than demand. The essential requirements of general anesthesia for IHD are avoiding tachycardia and extremes of blood pressure, both of which adversely affect the balance between oxygen supply and demand [7]. General anesthesia (GA) is associated with hypotension due to IV inducing agents; tachycardia and hypertension due to pressor response during direct laryngoscopy and tracheal intubation [8] leading to cardiac morbidity which did not occur in this case. GEA reduces preload and afterload, stress response, coagulation responses, post-operative analgesic requirement, and risk of perioperative MI [9]. There is evidence that homeostasis of the neuroendocrine system and hence the immune responses are better preserved after regional anesthesia than after general anesthesia [10].

Large doses of local anesthetic agents (LA) lead to myocardial toxicity and depression [11]. In this case, the drug was given slowly and at incremental doses with monitoring of hemodynamic parameters thereby preventing a sudden change in blood pressure and heart rate. Epidural LA along with opioids is better for postoperative analgesia. Yeager et al. concluded that epidural analgesia group patients had a lesser incidence of post-operative myocardial morbidity compared with GA alone for high-risk surgeries [12]. Beattie et al. in their meta-analysis on epidural analgesia concluded that reduction in cardiac events with post-operative epidural analgesia is important as it reduces post-operative MI also [13]. Rivers et al. compared EA with GA for infrainguinal arterial reconstruction and concluded that both regional and GA techniques remain equally acceptable [14]. 


\section{CONCLUSION}

GEA is a safe alternative to GA for anesthetic management in IHD patients coming for non-cardiac surgery. The advantage of GEA over GA is good analgesia and avoidance of pressor response to laryngoscopy, intubation, and extubation. It improves coronary function and myocardial oxygen balance in patients with ischemic heart disease, which results in increased myocardial performance and a reduction of the number and duration of ischemic episodes. Therefore, under close monitoring of cardiovascular status, graded epidural anesthesia is a safe and reliable technique for upper abdominal surgeries in elderly patients with ischemic heart disease.

\section{REFERENCES}

1. Writing Group Members, Mozaffarian D, Benjamin EJ, Go AS, Arnett DK, Blaha MJ, et al. Heart disease and stroke statistics-2016 update: A report from the American heart association. Circulation 2016;133:e38-60.

2. Fleisher LA, Fleischmann KE, Auerbach AD, Barnason SA, Beckman JA, Bozkurt B, et al. 2014 ACC/AHA guideline on perioperative cardiovascular evaluation and management of patients undergoing noncardiac surgery: Executive summary: A report of the American college of cardiology/ American heart association task force on practice guidelines. Circulation 2014;130:2215-45.

3. Kristensen SD, Knuuti J. New ESC/ESA guidelines on non-cardiac surgery: Cardiovascular assessment and management. Eur Heart J 2014;35:2344.

4. Liu S, Wu C. Effect of postoperative analgesia on major postoperative complications: A systematic update of the evidence. Anesth Analg 2007;104:689-702.
5. Peyton P, Myles P, Silbert B. Perioperative epidural analgesia and outcome after major abdominal surgery in high-risk patients. Anesth Analg 2003;96:548-54.

6. Guay J, Choi P, Suresh S. Neuraxial blockade for the prevention of postoperative mortality and major morbidity: An overview of Cochrane systemic reviews. Cochrane Database Syst Rev 2014;2014:CD010108.

7. Hedge J, Balajibabu PR, Sivaraman T. The patient with ischaemic heart disease undergoing noncardiac surgery. Indian J Anaesth 2017;61:705-11.

8. Hines AS, Marschall KE, editors. Ischemic Heart Disease Stoelting's Anesthesia and Co-Existing Disease. $5^{\text {th }}$ ed. Philadelphia, PA: ChurchillLivingstone; 2008. p. 17.

9. Blomberg S, Emanuelsson H, Ricksten SE. Thoracic epidural anesthesia and central hemodynamics in patients with unstable angina pectoris. Anesth Analg 1989;69:558-62.

10. Bernstein S, Malhotra V. Regional anesthesia for genitourinary surgery. In: Malhotra V, editor. Anesthesia for Renal Genito-Urologic Surgery. New York: McGraw-Hill; 1996. p. 265.

11. Kaul TK, Tayal G. Anaesthetic considerations in a cardiac patient undergoing noncardiac surgery. Indian J Anaesth 2007;51:280-6.

12. Yeager MP, Glass DD, Neff RK, Brinck-Johnsen T. Epidural anesthesia and analgesia in high risk surgical patients. Anesthesiology 1987;66:729-36.

13. Beattie WS, Badner NH, Choi P. Epidural analgesia reduces postoperative myocardial infarction: A metaanalysis. Anesth Analg 2001;93:853-8.

14. Rivers SP, Scher LA, Sheehan E, Veith FJ. Epidural versus general anesthesia for infrainguinal arterial reconstruction. J Vasc Surg 1991;14:764-8.

Funding: None; Conflicts of Interest: None Stated.

How to cite this article: Parveen S, Singh S. Graded Epidural Anesthesia for Open Cholecystectomy in Elderly Patient with Ischemic Heart Disease. Indian J Case Reports. 2021;7(10):452-454. 